\title{
3D Spectromicroscopic Observation of Yb-Silicate Ceramics Using XAFS-CT
}

\author{
Yasuo Takeichi $^{1,}{ }^{*}$, Toshiki Watanabe ${ }^{1}$, Yasuhiro $\mathrm{Niwa}^{1}$, Satoshi Kitaoka ${ }^{2}$ and Masao Kimura ${ }^{1}$ \\ 1. Institute of Materials Structure Science, High Energy Accelerator Research Organization (KEK), \\ Tsukuba, Japan. \\ 2. Japan Fine Ceramics Center, Nagoya, Japan. \\ * Corresponding author, yasuo.takeichi@kek.jp
}

Environmental barrier coatings (EBCs) are known to play an important role in enhancing the operating temperatures of gas-turbine engines [1]. A coating material in an operating engine undergoes various chemical reactions caused by oxygen, water vapor and other chemicals in the high-temperature combustion exhausts. Therefore, investigation of the chemical properties in the nanoscales is important to understand the degradation of EBCs. Here we report a result showing the 3D distribution of the chemical properties of ytterbium silicates $(\mathrm{Yb}-\mathrm{Si}-\mathrm{O})$ [2], a candidate for top-coat materials of next generation EBCs.

Ytterbium disilicate $\left(\mathrm{Yb}_{2} \mathrm{Si}_{2} \mathrm{O}_{7}\right)$ ceramics is reported to contain precipitates of $\mathrm{Yb}_{2} \mathrm{SiO}_{5}$, and the transition between $\mathrm{Yb}_{2} \mathrm{Si}_{2} \mathrm{O}_{7}$ and $\mathrm{Yb}_{2} \mathrm{SiO}_{5}$ is suggested to result from the chemical reactions during

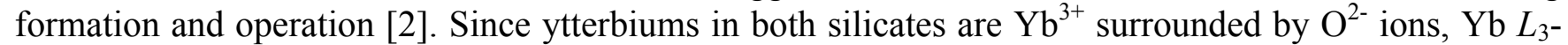
edge absorption spectra were only slightly different from each other. Therefore, this system would be a good test case to evaluate the spectroscopic capability of an X-ray microscope other than its importance as coatings; factors such as illumination homogeneity and stability, linearity of the detector, accuracy of the alignment and reconstruction, may affect the absorption spectra extracted from the 3D dataset.

A XAFS-CT instrument, an X-ray microscope combined with X-ray absorption fine structure, was recently installed at PF-AR NW2A beamline in KEK, Japan. The microscope (Xradia Ultra, Carl-Zeiss X-ray Microscopy, Inc.) was designed to work at the photon energy range of 5-11 keV. The illumination through the condenser capillary is homogenized using the agitators. The zone plate and optical magnification allows the projections of 20-40 $\mu \mathrm{m}$ FOV on the 12 -bit $2 \mathrm{k} \times 2 \mathrm{k}$ CCD detector. The spatial resolution was confirmed to be $<30 \mathrm{~nm}$ in the $2 \mathrm{D}$ observation. The magnification is maintained constant by moving the detector position along the X-ray beam axis depending on the photon energy.

The $\mathrm{Yb}-\mathrm{Si}-\mathrm{O}$ sample material was prepared as a sintered wafer by ultrasonic spray pyrolysis method [2]. The wafer was crushed to pick up a particle of 10-20 $\mu \mathrm{m}$ diameter for XAFS-CT observation. Projection images were obtained using a zone plate with the diameter and outermost zone width of $100 \mu \mathrm{m}$ and 30 nm, respectively. Each of the tomographies with 361 projections was repeated at 36 energy points in a range of 8900-9060 eV (around $\mathrm{Yb} L_{3}$-edge). Total aquisition time was about 12 hours. Alignment and reconstruction at each photon energy were performed using TXM Wizard software [3]. The voxel size was $24.4 \mathrm{~nm}$ for reconstruction, but was binned to $48.8 \mathrm{~nm}$ for segmentation described below.

The rendered 3D structure of $\mathrm{Yb}-\mathrm{Si}-\mathrm{O}$ sample observed at $8980 \mathrm{eV}$ is shown in Fig. 1(a). It was difficult to distinguish $\mathrm{Yb}_{2} \mathrm{Si}_{2} \mathrm{O}_{7}$ and $\mathrm{Yb}_{2} \mathrm{SiO}_{5}$ only using raw spectrum of each voxels, though island/sea structures with micropores were clearly observed in the 3D images. Therefore, we segmented the 3D images into high-density island and low-density sea regions. Morphological Segmentation plugin [4] of Fiji software package was used to segment the data. This plugin allows labeling and segmentation by the 
watershed analysis of the gradient of the absorption intensity. Figure 1(b) shows a slice of the segmented 3D image at the dotted line in Fig. 1(a). The segmentation successfully exploited grain/porous structures, while omitting the local fluctuations of the X-ray absorption possibly due to the projection and/or reconstruction artifacts.

Fig. 2 shows the averaged absorption spectra of the high-density island and low-density sea regions. Spectrum of each region was fitted by the linear combination of standard spectra, $\mathrm{Yb}_{2} \mathrm{Si}_{2} \mathrm{O}_{7}, \mathrm{Yb}_{2} \mathrm{SiO}_{5}$, and $\mathrm{Yb}_{2} \mathrm{O}_{3}$. The fitting range was limited to $8954-9040 \mathrm{eV}$ because the intensities at the white-line peak at around $8946 \mathrm{eV}$ showed some deviation, possibly due to large absorption. However, as shown in the inset of Fig. 2, a slight but significant difference in the fitting range quantified the fraction of the three standards. The high-density island region was found to contain $>70 \%$ of $\mathrm{Yb}_{2} \mathrm{SiO}_{5}$, while the low-density sea region was pure ( $>99 \%) \mathrm{Yb}_{2} \mathrm{Si}_{2} \mathrm{O}_{7}$. The amount of $\mathrm{Yb}_{2} \mathrm{O}_{3}$ was found to be negligible for both spectra. From this result we concluded that, from the spectroscopic point of view, the chemical state of $\mathrm{Yb}$ in island grains was identical to that of $\mathrm{Yb}_{2} \mathrm{SiO}_{5}$.

Moreover, these results tell that XAFS-CT observation is useful for evaluating not only the edge energy or valency, but also detailed X-ray absorption structures in the post-edge area. On the other hand, they have also shown the difficulty in obtaining a correct absorption spectrum, especially for materials whose spectrum has a sharp white-line peak, where the high linearity of the detector is required to cover a whole energy range of the absorption spectra [5].

\section{References:}

[1] D R Clarke and C G Levi, Annu. Rev. Mater. 33 (2003), 383.

[2] M Wada et al, Acta Mater. 135 (2017) 372.

[3] Y Liu et al., J. Synchrotron Rad. 19 (2012), 281.

[4] D Legland et al., Bioinfomatics 32 (2016), 3532.

[5] This work was supported by the Structural Materials for Innovation of the Cross ministerial Strategic Innovation Promotion Program (SM ${ }^{4}$ I, SIP) of Japan Science and Technology (JST). Experiments were performed under the approval of the Photon Factory Program Advisory Committee (Proposal Nos. 2015S2-002 and 2016S2-001).

(a)

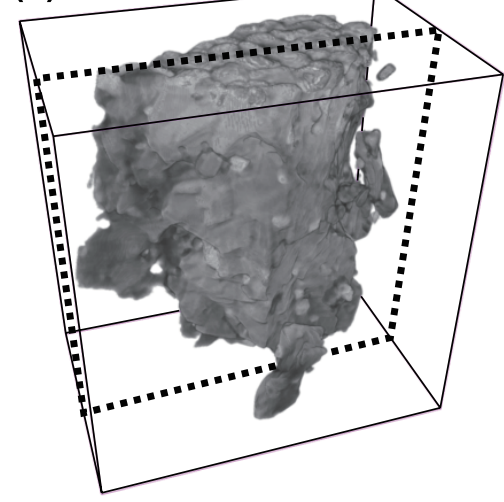

(b)

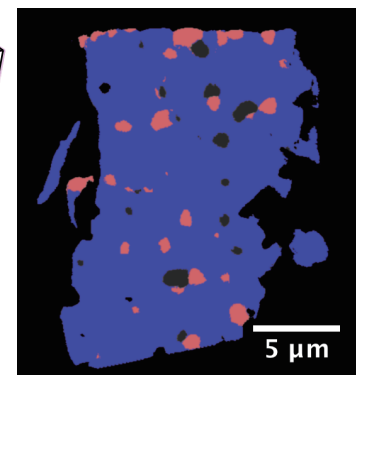

Figure. 1. (a) $3 \mathrm{D}$ view of a reconstructed $\mathrm{Yb}-\mathrm{Si}-\mathrm{O}$ sample observed at $8980 \mathrm{eV}$. (b) A slice of segmented dataset at dotted lines drawn in (a).

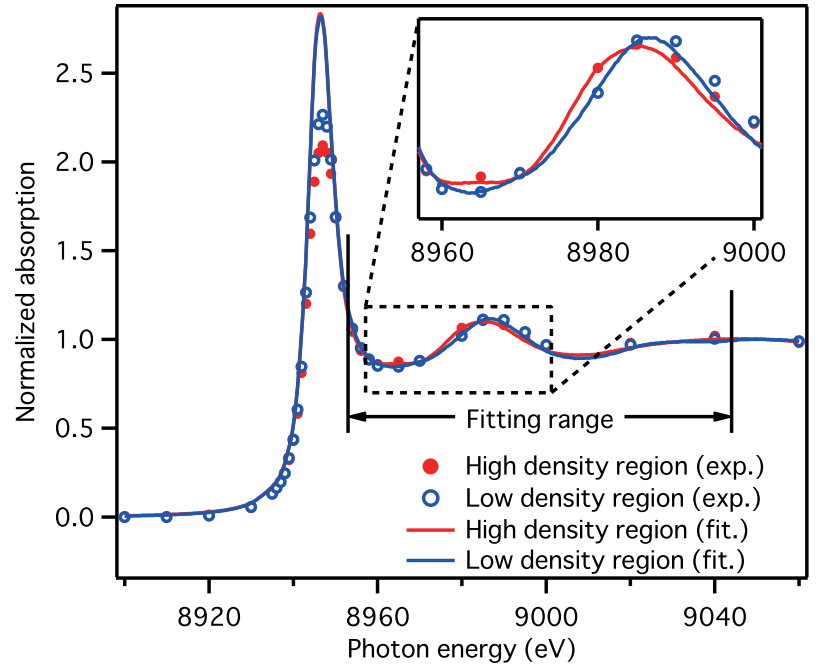

Figure. 2. $\mathrm{Yb} L_{3}$-edge $\mathrm{X}$-ray absorption spectra and fitting results of the red and blue regions shown in Fig. 1(b). 